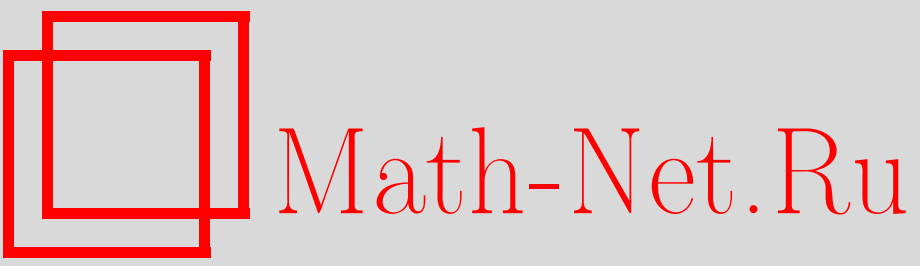

Ю. В. Павлов, Размерная регуляризация и $n$-волновая процедура для скалярных полей в многомерных квазиевклидовых пространствах, ТМФ, 2001, том 128, номер 2, 236-248

DOI: https://doi.org/10.4213/tmf494

Использование Общероссийского математического портала Math-Net.Ru подразумевает, что вы прочитали и согласны с пользовательским соглашением

http://www.mathnet.ru/rus/agreement

Параметры загрузки:

IP : 3.80 .253 .173

26 апреля 2023 г., 16:07:25 
ТЕОРЕТИЧЕСКАЯ

И МАТЕМАТИЧЕСКАЯ

ФИЗИКА

Том 128, № 2

август, 2001

(C) 2001 г.

Ю.В. Павлов*

\section{РАЗМЕРНАЯ РЕГУЛЯРИЗАЦИЯ И $n$-ВОЛНОВАЯ ПРОЦЕДУРА ДЛЯ СКАЛЯРНЫХ ПОЛЕЙ В МНОГОМЕРНЫХ КВАЗИЕВКЛИДОВЫХ ПРОСТРАНСТВАХ}

Получены выражения для вакуумных средних тензора энергии-импульса скалярного поля с произвольной связью с кривизной в $N$-мерном квазиевклидовом пространстве-времени для вакуума, определяемого методом диагонализации гамильтониана. $n$-Волновая процедура обобщена для многомерных пространств. Найдены все контрчлены для $N=5$ и для конформного скалярного поля в $N=6,7$. Определена геометрическая структура первых трех контрчленов для $N$-мерных пространств. Показано, что все вычитания в 4-мерном пространстве-времени и первые 3 вычитания в многомерных пространствах соответствуют перенормировке констант затравочного гравитационного лагранжиана. Обсуждается геометрическая структура других контрчленов в многомерном случае и вопрос об устранении конформной аномалии в 4-мерном пространстве-времени.

\section{1. ВВЕДЕНИЕ}

Обобщение результатов квантовой теории поля в искривленном пространстве-времени (см. монографии [1], [2]) на случай произвольной размерности может представлять самостоятельный интерес в связи с исследованиями многомерных теорий [3]. В то же время явная зависимость от размерности пространства в исследуемых выражениях необходима при последовательном проведении размерной регуляризации даже в случае 4-мерного пространства-времени.

Одним из центральных объектов квантовой теории в искривленном пространстве явлются вакуумные средние тензора энергии-импульса. В соответствии с полуклассическим подходом они являются источниками гравитационного поля в уравнениях Эйнштейна и их учет необходим при исследовании обратного влияния квантованного поля на геометрию пространства-времени. При их вычислении необходимо, во-первых, корректно определить вакуумное состояние и, во-вторых, устранить возникаюшие расходимости. Для скалярного поля с произвольной связью с кривизной определение вакуумного состояния, в соответствии с методом диагонализации гамильтониана [1], было дано в [4].

\footnotetext{
* Институт проблем машиноведения РАН, Санкт-Петербург, Россия. E-mail: pavlov@ipme.ru
} 
При устранении возникаюших расходимостей используются различные вычитательные процедуры (см. обзоры в [1], [2]). Наиболее эффективной в вычислительном плане является $n$-волновая процедура, предложенная в [5]. В работе [6] для 4-мерного случая была дана интерпретация всех бесконечных и части конечных вычитаний $n$-волновой процедуры в терминах перенормировок констант в затравочном лагранжиане гравитационного поля.

В случае размерности $N>4$ отсутствует (в нечетномерных пространствах) аналог теоремы Гаусса-Бонне и в квазиевклидовом случае нет линейной зависимости между тензорами, соответствуюшими вариационным производным квадратичных по скалярной кривизне и тензору Риччи слагаемых. Поэтому можно ожидать появления в затравочном гравитационном лагранжиане дополнительных по сравнению с 4-мерным случаем квадратичных по кривизне выражений.

В предлагаемой работе метод размерной регуляризации используется для выяснения геометрической интерпретации вычитаемых при помощи $n$-волновой процедуры выражений в квазиевклидовом $N$-мерном пространстве-времени. Рассматривается случай скалярного поля с произвольной связью с кривизной. Вакуумное состояние определяется в соответствии с методом диагонализации гамильтониана. В разделе 2 проведено квантование скалярного поля в квазиевклидовом пространстве и приведены выражения для вакуумных средних тензора энергии-импульса. В разделе 3 установлена структура расходимостей в $N$-мерном пространстве и представлены первые три контрчлена $n$-волновой процедуры для скалярного поля с произвольной связью с кривизной и контрчлены четвертого порядка для конформного поля. В разделе 4 даются сведения, необходимые для обобшения вычитательной процедуры на случай пространства-времени произвольной комплексной размерности $N-2 \varepsilon$, установлена геометрическая структура вычитаемых контрчленов и построен соответствуюший затравочный гравитационный лагранжиан. Обсуждается вопрос о поляризации вакуума и конформной аномалии в случае $N=4$. В приложении приведены выражения для встречаюшихся в работе геометрических величин в $N$-мерном пространстве-времени.

В статье используется система единиц, в которой $\hbar=c=1$.

\section{2. СКАЛЯРНОЕ ПОЛЕ В $N$-МЕРНОМ КВАЗИЕВКЛИДОВОМ ПРОСТРАНСТВЕ}

Рассмотрим комплексное скалярное поле $\phi(x)$ массы $m$ с лагранжианом

$$
L(x)=\sqrt{|g|}\left[g^{i k} \partial_{i} \phi^{*} \partial_{k} \phi-\left(m^{2}+\xi R\right) \phi^{*} \phi\right]
$$

и уравнением движения

$$
\left(\nabla^{i} \nabla_{i}+\xi R+m^{2}\right) \phi(x)=0
$$

где $\nabla_{i}$ - ковариантные производные в метрике $g_{i k}, g=\operatorname{det}\left(g_{i k}\right), R$ - скалярная кривизна, $\xi$ - константа связи с кривизной. В пространстве-времени размерности $N$ значение $\xi=\xi_{c}=(N-2) /[4(N-1)]$ соответствует конформной связи $\left(\xi_{c}=1 / 6\right.$ при $\left.N=4\right)$. 
При $\xi=\xi_{c}$ и $m=0$ уравнение (2) является конформно-инвариантным. Значение $\xi=0$ соответствует случаю минимальной связи.

Метрический тензор энергии-импульса (ТЭИ), получаемьй варьированием действия по метрике, имеет вид (см. [7])

$$
T_{i k}=\partial_{i} \phi^{*} \partial_{k} \phi+\partial_{k} \phi^{*} \partial_{i} \phi-g_{i k}|g|^{-\frac{1}{2}} L(x)-2 \xi\left[R_{i k}+\nabla_{i} \nabla_{k}-g_{i k} \nabla^{j} \nabla_{j}\right] \phi^{*} \phi
$$

где $R_{i k}$ - тензор Риччи.

Метрика $N$-мерного квазиевклидова пространства с одномерным временем и $(N-1)$ мерным плоским пространством может быть записана как

$$
d s^{2}=g_{i k} d x^{i} d x^{k}=d t^{2}-a^{2}(t) d \mathbf{x}^{2}=a^{2}(\eta)\left(d \eta^{2}-d \mathbf{x}^{2}\right)
$$

Полная система решений уравнения (2) в метрике (4) имеет вид

$$
\phi(x)=a^{-\frac{N-2}{2}}(\eta) g_{\lambda}(\eta) \Phi_{J}(\mathbf{x})
$$

где

$$
\begin{gathered}
\Phi_{J}(\mathbf{x})=(2 \pi)^{-\frac{N-1}{2}} \exp \left(-i \lambda_{\alpha} x^{\alpha}\right), \\
J=\left\{\lambda_{1}, \ldots, \lambda_{N-1}\right\}, \quad-\infty<\lambda_{\alpha}<+\infty, \quad \lambda=\left(\sum_{\alpha=1}^{N-1} \lambda_{\alpha}^{2}\right)^{\frac{1}{2}}, \\
g_{\lambda}^{\prime \prime}(\eta)+\Omega^{2}(\eta) g_{\lambda}(\eta)=0,
\end{gathered}
$$

штрих означает производную по конформному времени $\eta, \Omega(\eta)$ - осцилляторная частота,

$$
\Omega^{2}(\eta)=m^{2} a^{2}+\lambda^{2}-\Delta \xi a^{2} R, \quad \Delta \xi \equiv \xi_{c}-\xi .
$$

В соответствии с методом диагонализации гамильтониана [4] и с учетом нормировки $g_{\lambda} g_{\lambda}^{* \prime}-g_{\lambda}^{\prime} g_{\lambda}^{*}=-2 i$ функции $g_{\lambda}(\eta)$ должны удовлетворять начальным условиям

$$
g_{\lambda}^{\prime}\left(\eta_{0}\right)=i \Omega\left(\eta_{0}\right) g_{\lambda}\left(\eta_{0}\right), \quad\left|g_{\lambda}\left(\eta_{0}\right)\right|=\frac{1}{\sqrt{\Omega\left(\eta_{0}\right)}}
$$

Отметим, что используемые в [6] начальные условия соответствуют диагональной форме гамильтониана, только если $\xi=\xi_{c}$ или $R=0$.

Для квантования разложим поле $\phi(x)$ по полной системе решений уравнения (2):

$$
\phi(x)=\int d \mu(J)\left[\phi_{J}^{(+)} a_{J}^{(+)}+\phi_{J}^{(-)} a_{J}^{(-)}\right]
$$

где

$$
\phi_{J}^{(+)}(x)=\frac{a^{-\frac{N-2}{2}}(\eta)}{\sqrt{2}} g_{\lambda}(\eta) \Phi_{J}^{*}(\mathbf{x}), \quad \phi_{J}^{(-)}(x)=\left(\phi_{J}^{(+)}(\mathbf{x})\right)^{*}
$$


$d \mu(J)=\prod_{\alpha=1}^{N-1} d \lambda_{\alpha}$, и потребуем выполнения коммутационных соотношений

$$
\left[a_{J}^{(-)}, \stackrel{*}{a}_{J^{\prime}}^{(+)}\right]=\left[{ }_{a}^{*}(-), a_{J^{\prime}}^{(+)}\right]=\delta_{J J^{\prime}}, \quad\left[a_{J}^{( \pm)}, a_{J^{\prime}}^{( \pm)}\right]=\left[a_{J}^{*}( \pm), *_{J^{\prime}}^{( \pm)}\right]=0 .
$$

Средние от оператора ТЭИ по вакууму, уничтожаемому операторами $a_{J}^{(-)}$, удобно выражать через билинейные комбинации функций $g_{\lambda}$ и $g_{\lambda}^{*}$ :

$$
S=\frac{\left|g_{\lambda}^{\prime}\right|^{2}+\Omega^{2}\left|g_{\lambda}\right|^{2}}{4 \Omega}-\frac{1}{2}, \quad U=\frac{\Omega^{2}\left|g_{\lambda}\right|^{2}-\left|g_{\lambda}^{\prime}\right|^{2}}{2 \Omega}, \quad V=-\frac{d\left(g_{\lambda}^{*} g_{\lambda}\right)}{2 d \eta},
$$

которые в соответствии с (7) удовлетворяют системе дифференциальных уравнений

$$
S^{\prime}=\frac{\Omega^{\prime}}{2 \Omega} U, \quad U^{\prime}=\frac{\Omega^{\prime}}{\Omega}(1+2 S)-2 \Omega V, \quad V^{\prime}=2 \Omega U
$$

и начальным условиям, следуюшим из (9):

$$
S\left(\eta_{0}\right)=U\left(\eta_{0}\right)=V\left(\eta_{0}\right)=0
$$

Подставляя разложение (10) в (3) и проводя выгисления в $(N-1)$-мерных сферических координатах в пространстве $\left\{\lambda_{\alpha}\right\}$, получим для вакуумных средних ТЭИ следующие выражения:

$$
\left\langle 0\left|T_{i k}\right| 0\right\rangle=\frac{B_{N}}{a^{N-2}} \int_{0}^{\infty} d \lambda \lambda^{N-2} \tau_{i k},
$$

где $B_{N}=\left[2^{N-3} \pi^{(N-1) / 2} \Gamma((N-1) / 2)\right]^{-1}, \Gamma(z)$ - гамма-функция, а "спектральные плотности" $\tau_{i k}$ равны

$$
\begin{aligned}
\tau_{00}= & \Omega\left(S+\frac{1}{2}\right)+\Delta \xi(N-1)\left[c V+\left(c^{\prime}+(N-2) c^{2}\right) \frac{1}{\Omega}\left(S+\frac{1}{2} U+\frac{1}{2}\right)\right] \\
\tau_{\alpha \beta}= & \delta_{\alpha \beta}\left\{\frac{\lambda^{2}}{(N-1) \Omega}\left(S+\frac{1}{2}\right)-\frac{\Omega^{2}-\lambda^{2}}{2(N-1) \Omega} U-\right. \\
& \left.-\Delta \xi\left[(N-1) \frac{c^{\prime}}{\Omega}\left(S+\frac{1}{2} U+\frac{1}{2}\right)+2 \Omega U-(N-1) c V\right]\right\}
\end{aligned}
$$

и введено обозначение $c=a^{\prime} / a$. (При получении выражения для пространственных компонент (18) использованы уравнения движения.)

Приведем также формулу для следа вакуумного ТЭИ:

$$
\begin{aligned}
\left\langle 0\left|T_{i}^{i}\right| 0\right\rangle=\frac{B_{N}}{a^{N}} & \int_{0}^{\infty} d \lambda \lambda^{N-2}\left\{\frac{1}{\Omega}\left(S+\frac{1}{2} U+\frac{1}{2}\right) \times\right. \\
& \left.\times\left(m^{2} a^{2}+\Delta \xi(N-1)(N-2) c^{\prime}\right)+\Delta \xi(N-1)[2 \Omega U-(N-2) c V]\right\} .
\end{aligned}
$$

Отметим, что вакуумные средние ТЭИ, представленные в [1], [6], вычислены по вакуумному состоянию, не соответствующему (при $\xi \neq \xi_{c}$ и $R \neq 0$ ) диагональной форме гамильтониана, а представленные в [8] выражения для вакуумного ТЭИ справедливы только в размерности $N=4$. 


\section{3. РАСХОДИМОСТИ ТЭИ И $n$-ВОЛНОВАЯ ПРОЦЕДУРА}

Приведенные выражения для вакуумного ТЭИ расходятся на верхнем пределе по $\lambda$. Для анализа расходимостей запишем систему дифференциальных уравнений (14) с начальными условиями (15) в виде системы интегральных уравнений Вольтерра

$$
\begin{gathered}
U(\eta)+i V(\eta)=\int_{\eta_{0}}^{\eta} w\left(\eta_{1}\right)\left(1+2 S\left(\eta_{1}\right)\right) e^{2 i \Theta\left(\eta_{1}, \eta\right)} d \eta_{1}, \\
S(\eta)=\frac{1}{2} \int_{\eta_{0}}^{\eta} d \eta_{1} w\left(\eta_{1}\right) \int_{\eta_{0}}^{\eta_{1}} w\left(\eta_{2}\right)\left(1+2 S\left(\eta_{2}\right)\right) \cos \left[2 \Theta\left(\eta_{2}, \eta_{1}\right)\right] d \eta_{2},
\end{gathered}
$$

где $w(\eta)=\Omega^{\prime}(\eta) / \Omega(\eta), \Theta\left(\eta_{1}, \eta_{2}\right)=\int_{\eta_{1}}^{\eta_{2}} \Omega(\eta) d \eta$.

Используя первую итерацию в уравнениях $(20),(21)$ и учитывая, что $\Theta\left(\eta_{1}, \eta_{2}\right) \rightarrow$ $\lambda\left(\eta_{2}-\eta_{1}\right)$ при $\lambda \rightarrow \infty$, получим

$$
\begin{gathered}
U+i V \approx \int_{\eta_{0}}^{\eta} w\left(\eta_{1}\right) e^{2 i \lambda\left(\eta-\eta_{1}\right)} d \eta_{1}, \\
S \approx \frac{1}{4}\left|\int_{\eta_{0}}^{\eta} w\left(\eta_{1}\right) e^{2 i \lambda \eta_{1}} d \eta_{1}\right|^{2} .
\end{gathered}
$$

Поэтому $S \sim \lambda^{-6}, U \sim \lambda^{-4}, V \sim \lambda^{-3}$ при $\lambda \rightarrow \infty$. Из (17), (18) и свойств функций $S, U, V$ относительно замены $\lambda \rightarrow-\lambda$ следует, что в асимптотических разложениях $\tau_{i k}$ будут присутствовать только нечетные степени $\lambda$.

Таким образом, вакуумный ТЭИ (16) имеет в $N$-мерном квазиевклидовом пространстве $[N / 2]+1$ различных типов расходимостей ([b] обозначает целую часть числа $b$ ), которые растут как $\lambda^{N}, \lambda^{N-2}, \ldots, \ln \lambda$ при четном $N$ и как $\lambda^{N}, \lambda^{N-2}, \ldots, \lambda$ при нечетном $N$.

$n$-Волновая процедура, предложенная в [5], является одной из наиболее эффективных при вычислении перенормированных значений вакуумного ТЭИ:

$$
\left\langle 0\left|T_{i k}\right| 0\right\rangle_{\mathrm{ren}}=\frac{B_{N}}{a^{N-2}} \int_{0}^{\infty} d \lambda \lambda^{N-2} \tau_{i k}^{\mathrm{reg}},
$$

где

$$
\tau_{i k}^{\mathrm{reg}}=\tau_{i k}-\sum_{l=0}^{r} \tau_{i k}[l], \quad \tau_{i k}[l]=\frac{1}{l !} \lim _{n \rightarrow \infty} \frac{\partial^{l}}{\partial\left(n^{-2}\right)^{l}}\left(\frac{1}{n} \tau_{i k}(n \lambda, n m)\right),
$$

$r=[N / 2]$ в соответствии с проведенным подсчетом типов расходимостей.

Для получения явного вида контрчленов $\tau_{i k}[l]$ найдем разложение $S, U, V$ по обратным степеням $n$ при замене $\lambda \rightarrow n \lambda, m \rightarrow n m$ и $n \rightarrow \infty$ :

$$
S=\sum_{k=1}^{\infty} n^{-k} S_{k}, \quad U=\sum_{k=1}^{\infty} n^{-k} U_{k}, \quad V=\sum_{k=1}^{\infty} n^{-k} V_{k}
$$


Используя последовательные итерации в интегральных уравнениях (20), (21) и метод стационарной фазы, получим для первых отличных от нуля членов разложений

$$
\begin{gathered}
V_{1}=W, \quad U_{2}=D W, \quad S_{2}=\frac{1}{4} W^{2}, \quad V_{3}=\frac{1}{2} W^{3}-D^{2} W-\frac{\omega}{2} D\left(\frac{q}{\omega^{3}}\right) \\
U_{4}=\frac{3}{2} W^{2} D W-D^{3} W-D\left(\frac{\omega}{2} D\left(\frac{q}{\omega^{3}}\right)\right)+\frac{q}{2 \omega^{2}} D W \\
S_{4}=\frac{3}{16} W^{4}+\frac{1}{4}(D W)^{2}-\frac{1}{2} W D^{2} W-\frac{1}{4} \omega W D\left(\frac{q}{\omega^{3}}\right)
\end{gathered}
$$

где

$$
q=\Delta \xi a^{2} R, \quad \omega=\left(m^{2} a^{2}+\lambda^{2}\right)^{\frac{1}{2}}, \quad W=\frac{\omega^{\prime}}{2 \omega^{2}}, \quad D=\frac{1}{2 \omega} \frac{d}{d \eta}
$$

В случае конформного скалярного поля $(q=0)$ имеем

$$
\begin{gathered}
V_{5}=\frac{3}{8} W^{5}-\frac{5}{2} W(D W)^{2}-\frac{5}{2} W^{2} D^{2} W+D^{4} W \\
U_{6}=\frac{15}{8} W^{4} D W-\frac{5}{2}(D W)^{3}-10 W D W D^{2} W-\frac{5}{2} W^{2} D^{3} W+D^{5} W \\
S_{6}=\frac{5}{32} W^{6}-\frac{5}{8} W^{2}(D W)^{2}-\frac{5}{4} W^{3} D^{2} W-\frac{1}{2} D W D^{3} W+\frac{1}{2} W D^{4} W+\frac{1}{4}\left(D^{2} W\right)^{2}
\end{gathered}
$$

Необходимо отметить, что при вычислении величин $S_{k}, U_{k}, V_{k}$ мы, как и в [1], [5], [6], пренебрегаем зависящими от начального момента времени $\eta_{0}$ слагаемыми, т.е. ограничиваемся случаем контрчленов, локальных по времени $\eta$.

Используя (27)-(29), для величин $\tau_{i k}[l]$ получим

$$
\begin{gathered}
\tau_{00}[0]=\frac{\omega}{2}, \quad \tau_{\alpha \beta}[0]=\delta_{\alpha \beta} \frac{\lambda^{2}}{2(N-1) \omega}, \\
\tau_{00}[1]=\omega S_{2}+\Delta \xi(N-1)\left[c V_{1}+\frac{N-2}{4 \omega} c^{2}\right] \\
\tau_{\alpha \beta}[1]=\frac{\delta_{\alpha \beta}}{N-1}\left[\frac{\lambda^{2}}{\omega} S_{2}-\frac{m^{2} a^{2}}{2 \omega}\left(U_{2}+\frac{q}{2 \omega^{2}}\right)\right]+\Delta \xi\left[(N-1) c V_{1}-\right. \\
\left.-2 \omega\left(U_{2}+\frac{q}{2 \omega^{2}}\right)+\frac{1}{4 \omega}\left(-4 \xi a^{2} R+(N-1)(N-2) c^{2}\right)\right], \\
\tau_{00}[2]=\omega \\
\left.+S_{4}+\frac{q}{4 \omega^{2}} U_{2}+\frac{q^{2}}{16 \omega^{4}}\right)+ \\
\tau_{\alpha \beta}[2]= \\
\delta_{\alpha \beta}\left\{\frac { 1 } { N - 1 } \left[\frac{\lambda^{2}}{\omega}\left(S_{4}+\frac{q}{4 \omega^{2}} U_{2}+\frac{q^{2}}{16 \omega^{4}}\right)-\frac{m^{2} a^{2}}{2 \omega}\left(U_{4}+\frac{q^{2}}{4 \omega^{4}}+\right.\right.\right. \\
\left.\left.+\frac{q}{\omega^{2}} S_{2}\right)\right]+\Delta \xi\left[(N-1) c V_{3}-2 \omega\left(U_{4}+\frac{q^{2}}{4 \omega^{4}}+\frac{q}{\omega^{2}} S_{2}\right)+\right. \\
\left.\left.+\frac{1}{2 \omega}\left(-4 \xi a^{2} R+(N-1)(N-2) c^{2}\right)\left(S_{2}+\frac{1}{2} U_{2}+\frac{q}{4 \omega^{2}}\right)\right]\right\} .
\end{gathered}
$$

4 Теоретическая и математическая физика, т. 128, № 2, 2001 г. 
Из приведенных выражений видно, что структура контрчленов такая же, как и в [6], где при вычислении использованы начальные условия, отличные от (9). Это отмечено в [8] (для случая $N=4$ ) и согласуется с тем, что при вычислении $S_{k}, U_{k}, V_{k}$ не учитываются слагаемые, зависящие от начального момента времени $\eta_{0}$.

Заметим, что перенормированный ТЭИ ковариантно сохраняется. Это следует из равенства $\nabla^{i}\left(\tau_{i k}^{\text {reg }} / a^{N-2}\right)=0$, которое можно проверить, используя (34)-(38).

Формулы (34)-(38) определяют все контрчлены в случаях $N=4,5$. При $N \geqslant 6$ в соответствии с (25) имеются дополнительные контрчлены. Приведем их для $N=6,7$, ограничиваясь случаем конформного скалярного поля:

$$
\tau_{00}[3]=\omega S_{6}, \quad \tau_{\alpha \beta}[3]=\frac{\delta_{\alpha \beta}}{(N-1) \omega}\left[\lambda^{2} S_{6}-\frac{m^{2} a^{2}}{2} U_{6}\right] .
$$

\section{4. РАЗМЕРНАЯ РЕГУЛЯРИЗАЦИЯ И ГЕОМЕТРИЧЕСКАЯ СТРУКТУРА КОНТРЧЛЕНОВ}

Для установления геометрической структуры выражений, вычитаемых в соответствии с (24), (25) из вакуумного ТЭИ, проведем размерную регуляризацию, заменив $N$ на $N-2 \varepsilon$, где $\varepsilon-$ комплексный параметр. Тогда для размерно регуляризованных вычитаемых величин получим

$$
T_{i k, \varepsilon}[l]=\frac{B_{N-2 \varepsilon}}{a^{N-2}}(M a)^{2 \varepsilon} \int_{0}^{\infty} d \lambda \lambda^{N-2 \varepsilon} \tau_{i k, \varepsilon}[l],
$$

где $\tau_{i k, \varepsilon}[l]$ определяются по формулам (34)-(39) с заменой $N \rightarrow N-2 \varepsilon$, а $M$ - константа размерности массы, введенная для сохранения обычной размерности величин $T_{i k, \varepsilon}$ при $\varepsilon \neq 0[9]$.

Интегралы в (40) вычислим, используя равенство

$$
\int_{0}^{\infty} x^{k}\left(1+x^{2}\right)^{-p} d x=\frac{\Gamma\left(\frac{k+1}{2}\right) \Gamma\left(p-\frac{k+1}{2}\right)}{2 \Gamma(p)}
$$

и аналитическое продолжение выражения в правой части. В результате для 0-го и 1-го вычитаний получим выражения

$$
\begin{gathered}
T_{i k, \varepsilon}[0]=-\frac{m^{N}}{2^{N} \pi^{\frac{N}{2}}}\left(\frac{M^{2} 4 \pi}{m^{2}}\right)^{\varepsilon} \Gamma\left(\varepsilon-\frac{N}{2}\right) g_{i k} \\
T_{i k, \varepsilon}[1]=\frac{m^{N-2}}{2^{N-1} \pi^{\frac{N}{2}}}\left(\frac{1}{6}-\xi\right)\left(\frac{M^{2} 4 \pi}{m^{2}}\right)^{\varepsilon} \Gamma\left(1-\frac{N}{2}+\varepsilon\right) G_{i k}=\frac{m^{N-2}}{2^{N-1} \pi^{\frac{N}{2}}} \times \\
\times\left(\frac{M^{2} 4 \pi}{m^{2}}\right)^{\varepsilon}\left[\frac{-\Gamma\left(3-\frac{N}{2}+\varepsilon\right)}{3(N-1-2 \varepsilon)(N-2-2 \varepsilon)}+\Delta \xi \Gamma\left(1-\frac{N}{2}+\varepsilon\right)\right] G_{i k},
\end{gathered}
$$

где $G_{i k}$ - тензор Эйнштейна. 
Общие формулы (42), (43) при $N=4, \varepsilon \rightarrow 0$ соответствуют выражениям (50), (51), приведенным в [6] (дополнительный множитель 2 в нашем случае объясняется тем, что мы рассматриваем комплексное скалярное поле). При $N-2 \varepsilon=5$ имеем

$$
T_{i k, \varepsilon}[0]=\frac{m^{5}}{60 \pi^{2}} g_{i k}, \quad T_{i k, \varepsilon}[1]=-\frac{m^{3}}{576 \pi^{2}}(1-48 \Delta \xi) G_{i k} .
$$

Для $N=6$, сохраняя только ненулевые при $\varepsilon \rightarrow 0$ слагаемые, получим

$$
\begin{gathered}
T_{i k, \varepsilon}[0]=\frac{m^{6}}{384 \pi^{3}}\left(\frac{1}{\varepsilon}+b-\frac{1}{6}\right) g_{i k}, \\
T_{i k, \varepsilon}[1]=-\frac{m^{4}}{1920 \pi^{3}}\left[\frac{1}{\varepsilon}+b-\frac{11}{10}-30 \Delta \xi\left(\frac{1}{\varepsilon}+b-\frac{1}{2}\right)\right] G_{i k},
\end{gathered}
$$

где $b=2-\ln \left(m^{2} /\left(4 \pi M^{2}\right)\right)-C, C$ - постоянная Эйлера.

Из выражений (42), (43) следует, что для квазиевклидова пространства произвольной размерности $N$ 0-е вычитание в $n$-волновой процедуре соответствует перенормировке космологической постоянной, а 1-е вычитание - перенормировке гравитационной постоянной.

Выяснение геометрической структуры контрчлена $T_{i k, \varepsilon}[2]$ представляет значительные вычислительные трудности. Из (37), (38) можно установить, что $T_{i k, \varepsilon}[2]$ представим в виде произведения $m^{N-4}$ на множитель размерности (длина) $)^{-2}$, который является ковариантно сохраняюшимся тензором (в силу явно проверяемого ковариантного сохранения $\left.T_{i k, \varepsilon}[2]\right)$. Будем искать такой тензор в виде линейной комбинации вариационных производных (деленных на $\sqrt{|g|}$ ) от квадратичных по кривизне выражений (см. [10]):

$$
\begin{aligned}
{ }^{(1)} H_{i k}= & \frac{\delta \int d^{N} x \sqrt{|g|} R^{2}}{\sqrt{|g|} \delta g^{i k}}=2\left(\nabla_{i} \nabla_{k} R-g_{i k} \nabla^{l} \nabla_{l} R\right)+2 R\left(R_{i k}-\frac{1}{4} R g_{i k}\right), \\
{ }^{(2)} H_{i k}= & \frac{1}{\sqrt{|g|}} \frac{\delta}{\delta g^{i k}} \int d^{N} x \sqrt{|g|} R^{l m} R_{l m}=\nabla_{i} \nabla_{k} R-\nabla^{l} \nabla_{l} R_{i k}- \\
& -\frac{1}{2}\left(\nabla^{l} \nabla_{l} R+R^{l m} R_{l m}\right) g_{i k}+2 R^{l m} R_{l i m k}, \\
H_{i k}= & \frac{1}{\sqrt{|g|}} \frac{\delta}{\delta g^{i k}} \int d^{N} x \sqrt{|g|} R^{i k l m} R_{i k l m}=2 \nabla_{i} \nabla_{k} R-4 \nabla^{l} \nabla_{l} R_{i k}- \\
& -4 R_{i l} R_{k}^{l}+4 R^{l m} R_{l i m k}-\frac{1}{2} g_{i k} R_{l m p q} R^{l m p q}+2 R_{i l m p} R_{k}^{l m p} .
\end{aligned}
$$

Данные выражения имеют требуемую размерность (длина $)^{-2}$. Обозначим

$$
A_{i k}={ }^{(2)} H_{i k}-\frac{N}{4(N-1)}{ }^{(1)} H_{i k}
$$

Используя формулы приложения (П.6)-(П.18), можно убедиться, что в квазиевклидовом пространстве

$$
H_{i k}=\frac{4}{N-2} A_{i k}+\frac{1}{N-1}{ }^{(1)} H_{i k}
$$


Таким образом, $T_{i k, \varepsilon}[2]$ есть линейная комбинация двух тензоров ${ }^{(1)} H_{i k}$ и $A_{i k}$ :

$$
\begin{aligned}
& T_{i k, \varepsilon}[2]=\frac{m^{N-4}}{2^{N} \pi^{\frac{N}{2}}}\left(\frac{M^{2} 4 \pi}{m^{2}}\right)^{\varepsilon}\left[-\frac{2 \Gamma\left(4-\frac{N}{2}+\varepsilon\right)}{45(N-2-2 \varepsilon)(N-4-2 \varepsilon)} A_{i k}+\right. \\
& \left.\quad+\left(\frac{\Gamma\left(4-\frac{N}{2}+\varepsilon\right)}{60(N-1-2 \varepsilon)^{2}}+\Delta \xi \frac{\Gamma\left(3-\frac{N}{2}+\varepsilon\right)}{3(N-1-2 \varepsilon)}+(\Delta \xi)^{2} \Gamma\left(2-\frac{N}{2}+\varepsilon\right)\right){ }^{(1)} H_{i k}\right]
\end{aligned}
$$

Для вычисления предела $T_{i k, \varepsilon}[2]$ при $N=4, \varepsilon \rightarrow 0$ воспользуемся равенством, которое можно проверить с помошью формул (П.17), (П.18):

$$
{ }^{(3)} H_{i k}=\lim _{N \rightarrow 4} \frac{2(N-3)}{N-4} A_{i k}
$$

где ${ }^{(3)} H_{i k}$ - тензор, ковариантно сохраняющийся в 4-мерном конформно-плоском пространстве [11],

$$
{ }^{(3)} H_{i k}=R_{i}^{l} R_{l k}-\frac{2}{3} R R_{i k}-\frac{1}{2}\left(R^{l m} R_{l m}-\frac{1}{2} R^{2}\right) g_{i k} .
$$

Сохраняя ненулевые при $\varepsilon \rightarrow 0$ слагаемые, в итоге получим

$$
T_{i k}[2]=\frac{1}{1440 \pi^{2}}\left[-{ }^{(3)} H_{i k}+\left(\frac{1}{6}+10 \Delta \xi+90(\Delta \xi)^{2}\left(\frac{1}{\varepsilon}+b-2\right)\right){ }^{(1)} H_{i k}\right],
$$

что соответствует результату [6]. Однако необходимо отметить, что в размерности $4-2 \varepsilon$ $\Delta \xi=(1-\varepsilon)(6-4 \varepsilon)^{-1}-\xi$ и замена $\Delta \xi \rightarrow 6^{-1}-\xi$, используемая в [6], неверна из-за наличия множителя $(1 / \varepsilon)$.

При $N-2 \varepsilon=5$ имеем

$$
T_{i k}[2]=\frac{m}{\pi^{2} 2^{4}}\left[-\frac{1}{270} A_{i k}+\left(\frac{1}{3840}+\frac{\Delta \xi}{24}-(\Delta \xi)^{2}\right)^{(1)} H_{i k}\right] .
$$

В случае $N=6, \varepsilon \rightarrow 0$

$$
T_{i k}[2]=\frac{m^{2}}{\pi^{3} 2^{6}}\left[-\frac{A_{i k}}{180}+\left(\frac{1}{1500}+\frac{\Delta \xi}{15}\left(\frac{1}{\varepsilon}+b-2\right)-(\Delta \xi)^{2}\left(\frac{1}{\varepsilon}+b-1\right)\right){ }^{(1)} H_{i k}\right] .
$$

Из выражения (52) следует, что в $N$-мерном квазиевклидовом пространстве вычитание $T_{i k, \varepsilon}[2]$ соответствует перенормировке констант при $R^{2}$ и $R^{i k} R_{i k}$ в затравочном гравитационном лагранжиане.

Таким образом, в квазиевклидовом пространстве размерности $N$ вычитание контрчленов $T_{i k, \varepsilon}[0], T_{i k, \varepsilon}[1], T_{i k, \varepsilon}[2]$ может интерпретироваться в терминах перенормировки констант затравочного гравитационного лагранжиана вида

$$
L_{g r, 0}=\sqrt{|g|}\left[\frac{1}{16 \pi G_{0}}\left(R-2 \Lambda_{0}\right)+\alpha_{0}\left(R^{i k} R_{i k}-\frac{N R^{2}}{4(N-1)}\right)+\beta_{0} R^{2}\right] .
$$


Подчеркнем, что выражение (58) остается верным и при $N \rightarrow 4$, но в этом случае константа $\alpha_{0}$ испытывает в соответствии с (52) бесконечную перенормировку, пропорциональную $(N-4)^{-1}$. В 4-мерном случае бесконечна перенормировка и других констант: космологической $\Lambda_{0}$ и, для неконформного поля, гравитационной $G_{0}$ и константы $\beta_{0} . \mathrm{B}$ квантовой теории поля бесконечная перенормировка констант затравочного лагранжиана является стандартной ситуацией. Поэтому можно утверждать, что сделанный в [6] вывод о несоответствии контрчленов, пропорциональных ${ }^{(3)} H_{i k}$ (см. $\left.(55)\right)$, какой-либо перенормировке, является необоснованным, а их интерпретация как “истинной" поляризации вакуума весьма условна.

В пространствах с $N \geqslant 6$ необходимо учитывать, в соответствии с $n$-волновой процедурой $(24),(25)$, дополнительные по сравнению с 4-мерным случаем контрчлены (для конформного поля в $N=6,7$ они указаны в $(39))$. Вопрос об их геометрической структуре остается нерешенным. Исходя из соображений размерности и наличия высших производных от метрики в контрчленах можно утверждать, что в затравочном гравитационном лагранжиане в случае многомерных пространств с $N \geqslant 6$ должны присутствовать скалярные выражения с третьей и более высокими степенями кривизны, тензора Риччи и их ковариантными производными. Например, исходя из (31)-(33) можно ожидать для $N=6,7$ появления в затравочном лагранжиане слагаемых вида $\gamma R^{3}, \nu R R_{i}^{k} R_{k}^{i}$, $\mu R_{i}^{l} R_{l}^{k} R_{k}^{i}, \rho \nabla^{l} R \nabla_{l} R, \zeta R \nabla^{i} \nabla^{k} R_{i k}$ и т.д. В связи с этим отметим аналогию перенормировки в многомерном пространстве со случаем радиационных поправок высших порядков в 4-мерном пространстве-времени (см., например, [12]).

Приведем также вид первых трех контрчленов для следа вакуумного ТЭИ в $N$-мерном квазиевклидовом пространстве:

$$
\begin{aligned}
T_{i, \varepsilon}^{i}[0]=\frac{m^{N}}{2^{N-1} \pi^{\frac{N}{2}}}\left(\frac{M^{2} 4 \pi}{m^{2}}\right)^{\varepsilon} \Gamma\left(1-\frac{N}{2}+\varepsilon\right), \\
T_{i, \varepsilon}^{i}[1]=\frac{m^{N-2}}{2^{N-1} \pi^{\frac{N}{2}}}\left(\frac{M^{2} 4 \pi}{m^{2}}\right)^{\varepsilon}\left[\frac{\Gamma\left(3-\frac{N}{2}+\varepsilon\right)}{6(N-1-2 \varepsilon)}+\Delta \xi \Gamma\left(2-\frac{N}{2}+\varepsilon\right)\right] R, \\
T_{i, \varepsilon}^{i}[2]=\frac{m^{N-4}}{2^{N} \pi^{\frac{N}{2}}}\left(\frac{M^{2} 4 \pi}{m^{2}}\right)^{\varepsilon}\left\{\frac { \Gamma ( 4 - \frac { N } { 2 } + \varepsilon ) } { 9 0 } \left[\frac{2}{N-2-2 \varepsilon} R^{l m} R_{l m}-\right.\right. \\
\left.-\frac{5(N-2 \varepsilon)^{2}-20(N-2 \varepsilon)+24}{4(N-1-2 \varepsilon)^{2}(N-2-2 \varepsilon)} R^{2}-\frac{3}{N-1-2 \varepsilon} \square R\right]- \\
-\left(\Delta \xi \frac{\Gamma\left(3-\frac{N}{2}+\varepsilon\right)}{3(N-1-2 \varepsilon)}+(\Delta \xi)^{2} \Gamma\left(2-\frac{N}{2}+\varepsilon\right)\right) \times \\
\left.\times\left(2(N-1-2 \varepsilon) \square R+\frac{1}{2}(N-4-2 \varepsilon) R^{2}\right)\right\}
\end{aligned}
$$

где $\square R \equiv \nabla^{l} \nabla_{l} R$. При $N=4, \varepsilon \rightarrow 0$ формулы (59)-(61) соответствуют выражениям $(56)-(58)$ в $[6]$.

Обсудим вопрос об устранении аномального следа ТЭИ перенормировкой констант затравочного гравитационного лагранжиана. Аномальный след ТЭИ в 4-мерном про- 
странстве-времени может быть получен из $(61)$ при $N=4, \Delta \xi=0, \varepsilon \rightarrow 0$. Из равенств (П.19), (П.20) и формулы (52) следует, что конформная аномалия может быть устранена перенормировкой константы $\beta_{0}$ (конечной для конформного поля) и константы $\alpha_{0}$ (бесконечной, расходяшейся как $(N-4)^{-1}$ ) в затравочном лагранжиане (58). Однако возможность устранения аномальных слагаемых не означает их отсутствия для квантованного поля в искривленном пространстве. Это может быть выяснено только с помошью эксперимента (см. также обсуждение вопроса об устранении из аномального следа слагаемого, пропорционального $\square R$ в [2], §6.3).

\section{5. ЗАКЛЮЧЕНИЕ}

В данной работе исследовалась геометрическая структура контрчленов для вакуумного ТЭИ скалярного поля с произвольной связью с кривизной в $N$-мерном квазиевклидовом пространстве-времени. Полученные формулы (16)-(18) определяют неперенормированные вакуумные средние ТЭИ в $N$-мерном пространстве по вакууму, определяемому методом диагонализации гамильтониана [4]. Для устранения расходимостей ТЭИ используется $n$-волновая процедура [5], которая, в соответствии с проведенным анализом расходимостей, обобщена на случай $N$-мерных пространств. При этом появляются дополнительные по сравнению с 4-мерным случаем контрчлены. В работе они приведены для конформного поля в $N=6,7$ (см. (39)). Как видно из (31)-(33), даже для конформного скалярного поля вычисление перенормированного ТЭИ значительно усложняется по сравнению с 4-мерным случаем. Поэтому применение $n$-волновой процедуры в многомерных пространствах становится чрезвычайно трудоемким.

С помощью размерной регуляризации в работе найдена геометрическая структура трех первых контрчленов (42), (43), (52) n-волновой процедуры для вакуумных средних ТЭИ и (см. (59)-(61)) для следа вакуумного ТЭИ. Последовательное проведение вычислений в $N$-мерном пространстве позволило сделать вывод, что с использованием размерной регуляризации все вычитания $n$-волновой процедуры в 4-мерном пространстве можно интерпретировать в терминах перенормировок констант затравочного гравитационного лагранжиана (58). Данный результат противоречит утверждению [6], где такая интерпретация исключалась для конечных вычитаний, пропорциональных тензору ${ }^{(3)} H_{i k}$. В то же время в данной работе предельные выражения для $T_{i k, \varepsilon}[2]$ при $N=4$, $\varepsilon \rightarrow 0$ соответствуют результату в [6]. Парадоксальность ситуации разрешается равенством (53), которое показывает, что в квазиевклидовом пространстве тензор ${ }^{(3)} H_{i k}$ является пределом (по размерности) аналитически продолженного по размерности тензора (50), являющегося линейной комбинацией вариационных производных (47), (48) от выражений, квадратичных по скалярной кривизне и тензору Риччи.

Интерпретация всех конечных вычитаний $n$-волновой процедуры в 4 -мерном пространстве как перенормировок констант затравочного гравитационного лагранжиана позволяет сделать вывод о возможности их устранения такой перенормировкой. Аналогична ситуация с конформной аномалией следа вакуумного ТЭИ. Отметим, что физические значения соответствуюших констант могут быть определены только из эксперимента. 
Согласно $n$-волновой процедуре в $N$-мерном пространстве возникают дополнительные контрчлены, которые содержат максимальную степень производной от метрики, равную $2[N / 2]$. В этом можно убедиться, рассматривая асимптотические разложения (26) решений интегральных уравнений (20), (21). Поэтому при исследовании квантованных полей в искривленном $N$-мерном пространстве в затравочном гравитационном лагранжиане следует рассматривать комбинации геометрических величин, содержашие производные от метрики порядка $2([N / 2]-1)$. В задачах об обратном влиянии квантованного поля на метрику $N$-мерного пространства возникают, следовательно, дифференциальные уравнения для метрики порядка $2[N / 2]$.

ПРИЛОЖЕНИЕ

Приведем выражения для некоторых геометрических величин в $N$-мерном пространстве с метрикой (4).

Символы Кристоффеля, отличные от 0:

$$
\Gamma_{00}^{0}=\frac{a^{\prime}}{a} \equiv c, \quad \Gamma_{0 \beta}^{\alpha}=\Gamma_{\alpha \beta}^{0}=c \delta_{\alpha \beta} .
$$

Компоненты тензора $R_{i j k l}=g_{i m}\left(\partial_{l} \Gamma_{j k}^{m}-\partial_{k} \Gamma_{j l}^{m}+\Gamma_{n l}^{m} \Gamma_{j k}^{n}-\Gamma_{n k}^{m} \Gamma_{j l}^{n}\right):$

$$
R_{0 \alpha 0 \beta}=-a^{2} c^{\prime} \delta_{\alpha \beta}, \quad R_{\alpha \beta \gamma \nu}=a^{2} c^{2}\left(\delta_{\alpha \gamma} \delta_{\beta \nu}-\delta_{\alpha \nu} \delta_{\beta \gamma}\right) .
$$

Компоненты тензора Риччи, отличные от нуля, и скалярная кривизна:

$$
\begin{gathered}
R_{00}=(N-1) c^{\prime}, \quad R_{\alpha \beta}=-\delta_{\alpha \beta}\left[c^{\prime}+(N-2) c^{2}\right], \\
R=a^{-2}(N-1)\left[2 c^{\prime}+(N-2) c^{2}\right] .
\end{gathered}
$$

Компоненты тензора Эйнштейна $G_{i k}=R_{i k}-R g_{i k} / 2$ :

$$
G_{00}=-(N-1)(N-2) \frac{c^{2}}{2}, \quad G_{\alpha \beta}=\delta_{\alpha \beta}(N-2)\left[c^{\prime}+(N-3) \frac{c^{2}}{2}\right] .
$$

Используя (П.1)-(П.4), получим для квадратичных по кривизне выражений и вторых производных

$$
\begin{aligned}
R_{l m} R^{l m}= & a^{-4}(N-1)\left[N c^{\prime 2}+2(N-2) c^{\prime} c^{2}+(N-2)^{2} c^{4}\right], \\
\nabla^{l} \nabla_{l} R= & a^{-4} 2(N-1)\left[c^{(3)}+2(N-4) c^{\prime \prime} c+(N-4) c^{\prime 2}+\right. \\
& \left.+\left(N^{2}-11 N+22\right) c^{\prime} c^{2}-(N-2)(N-4) c^{4}\right], \\
\nabla^{l} \nabla_{l} R_{00}= & a^{-2}(N-1)\left[c^{(3)}+(N-6) c^{\prime \prime} c-2 c^{\prime 2}-\right. \\
& \left.-4(N-3) c^{\prime} c^{2}+2(N-2) c^{4}\right], \\
\nabla^{l} \nabla_{l} R_{\alpha \beta}= & -\delta_{\alpha \beta} a^{-2}\left[c^{(3)}+(3 N-10) c^{\prime \prime} c+2(N-3) c^{\prime 2}+\right. \\
& \left.+2\left(N^{2}-9 N+16\right) c^{\prime} c^{2}-2(N-2)(N-3) c^{4}\right],
\end{aligned}
$$




$$
\begin{aligned}
R^{l m} R_{l 0 m 0} & =a^{-2}(N-1)\left[c^{\prime 2}+(N-2) c^{\prime} c^{2}\right], \\
R^{l m} R_{l \alpha m \beta} & =-\delta_{\alpha \beta} a^{-2}\left[(N-1) c^{\prime 2}+(N-2) c^{\prime} c^{2}+(N-2)^{2} c^{4}\right], \\
R_{0 l m p} R_{0}^{l m p} & =a^{-2} 2(N-1) c^{\prime 2}, \\
R_{\alpha l m p} R_{\beta}^{l m p} & =-\delta_{\alpha \beta} a^{-2} 2\left[c^{\prime 2}+(N-2) c^{4}\right], \\
R_{l m p q} R^{l m p q} & =a^{-4} 2(N-1)\left[2 c^{\prime 2}+(N-2) c^{4}\right] .
\end{aligned}
$$

Для компонент вариационных производных (47), (50) получим, используя (П.6)-(П.11),

$$
\begin{aligned}
{ }^{(1)} H_{00}= & \frac{(N-1)^{2}}{a^{2}}\left[2 c^{\prime 2}-4 c^{\prime \prime} c-4(N-4) c^{\prime} c^{2}-\frac{c^{4}}{2}(N-2)(N-10)\right], \\
{ }^{(1)} H_{\alpha \beta}= & \delta_{\alpha \beta} a^{-2}(N-1)\left[4 c^{(3)}+4(2 N-9) c^{\prime \prime} c+2(3 N-11) c^{\prime 2}+\right. \\
& \left.+6\left(N^{2}-10 N+20\right) c^{\prime} c^{2}+\frac{1}{2}(N-2)\left(N^{2}-15 N+50\right) c^{4}\right], \\
A_{00}= & a^{-2} 8^{-1}(N-1)(N-2)^{2}(N-4) c^{4}, \\
A_{\alpha \beta}= & -\delta_{\alpha \beta} a^{-2} 8^{-1}(N-2)^{2}(N-4)\left[4 c^{\prime} c^{2}+(N-5) c^{4}\right] .
\end{aligned}
$$

Из (47), (48), (50) могут быть получены следующие выражения для следов:

$$
\begin{aligned}
{ }^{(1)} H_{i}^{i} & =-\frac{1}{2}(N-4) R^{2}-2(N-1) \nabla^{i} \nabla_{i} R, \\
A_{i}^{i} & =\frac{N-4}{2}\left[\frac{N}{4(N-1)} R^{2}-R^{l m} R_{l m}\right] .
\end{aligned}
$$

Благодарности. Автор благодарит проф̆. А. А. Гриба и участников семинара лаборатории теоретической физики им. А. А. Фридмана за обсуждение работы.

\section{Список литературы}

[1] А.А. Гриб, С. Г. Мамаев, В. М. Мостепаненко. Вакуумные квантовые эффекты в сильных полях. М.: Энергоатомиздат, 1988.

[2] Н. Биррелл, П. Девис. Квантованные поля в искривленном пространстве-времени. М.: Мир, 1984.

[3] Ю. С. Владимиров. Размерность физического пространства-времени и объединение взаимодействий. М.: Изд-во МГУ, 1987.

[4] Ю. В. Павлов. ТМФ. 2001. Т. 126. № 1. С. 115.

[5] Я.Б. Зельдович, А.А. Старобинский. ЖЭТФ. 1971. Т. 61. С. 2161.

[6] С. Г. Мамаев, В. М. Мостепаненко, В. А. Шелюто. ТМФ. 1985. Т. 63. № 1. С. 64.

[7] N. A. Chernikov, E. A. Tagirov. Ann. Inst. H. Poincaré. A. 1968. V. 9. P. 109.

[8] M. Bordag, J. Lindig, V. M. Mostepanenko. Class. Quantum Grav. 1998. V. 15. P. 581.

[9] G.t'Hooft, M. Veltman. Nucl. Phys. B. 1972. V. 44. P. 189.

[10] Б. С. Девитт. Динамическая теория групп и полей. М.: Наука, 1987.

[11] В. Л. Гинзбург, Д. А. Кирэнии, А. А. Любушин. ЖЭТФ. 1971. Т. 60. С. 451.

[12] В. А. Бейлин, Г. М. Верешков, Ю. С. Гришкан и др. ЖЭТФ. 1980. Т. 78. С. 2081.

Поступила в редакцию 13.XII.2000 г. 